\title{
Quantum Enhancements and Biquandle Brackets
}

\author{
Sam Nelson* $\quad$ Michael E. Orrison ${ }^{\dagger} \quad$ Veronica Rivera ${ }^{\ddagger}$
}

\begin{abstract}
We introduce a new class of quantum enhancements we call biquandle brackets, which are customized skein invariants for biquandle colored links. Quantum enhancements of biquandle counting invariants form a class of knot and link invariants that includes biquandle cocycle invariants and skein invariants such as the HOMFLY-PT polynomial as special cases, providing an explicit unification of these apparently unrelated types of invariants. We provide examples demonstrating that the new invariants are not determined by the biquandle counting invariant, the knot quandle, the knot group or the traditional skein invariants.
\end{abstract}

KEYWORDS: biquandles, biquandle brackets, quantum invariants, quantum enhancements of counting invariants

2010 MSC: 57M27, 57M25

\section{Introduction}

Biquandles, algebraic structures with axioms derived from the Reidemeister moves for oriented knots, were introduced in $[8$ and have been used to define invariants of oriented knots and links in [7, 12] and more. In particular, the number of biquandle colorings of an oriented knot or link diagram $K$ by a finite biquandle $X$ defines a nonnegative integer-valued invariant known as the biquandle counting invariant, denoted $\Phi_{X}^{\mathbb{Z}}(K)$. An enhancement of $\Phi_{X}^{\mathbb{Z}}$ is a generally stronger invariant from which $\Phi_{X}^{\mathbb{Z}}$ can be recovered; enhancements have been studied in [2, 4, 10, 15, to name just a few.

In [14] the first and last listed authors introduced the notion of quantum enhancements of $\Phi_{X}^{\mathbb{Z}}$ defined as quantum invariants of biquandle-colored knot or link diagrams, focusing on the unoriented case. In this paper we introduce a new infinite family of quantum enhancements using biquandle brackets, i.e., skein relations which depend on biquandle colorings. This family of invariants includes biquandle counting invariants, biquandle (and quandle) cocycle invariants, and classical quantum invariants such as the Jones and HOMFLYPT polynomials (see for example [13]) as special cases. In particular, we provide examples of strongly heterogeneous quantum enhancements, i.e., solutions to the biquandle-colored Yang-Baxter equation which are not solutions to the uncolored Yang-Baxter equation, settling a question from [14] and confirming that there are quantum enhancements which are neither cocycle invariants nor classical skein invariants.

The biquandle bracket conditions we find are very similar to the biquandle 2-cocycle condition, and indeed biquandle 2-cocycle invariants form a special case of biquandle brackets. Moreover, we identify an equivalence relation on biquandle brackets yielding the same invariant which specializes to the cohomology relation for biquandle cocycles, even for non-cocycle biquandle brackets. Connections between quantum invariants and quandle cocycle invariants were also studied in 9 .

The paper is organized as follows. In Section 2 we review the basics of biquandles and the biquandle counting invariant. In Section 3 we define biquandle brackets and provide some examples, including as an application a new skein invariant with values in the Galois field of eight elements $\mathbb{F}_{8}$. In Section 4 we consider the special case of biquandle brackets when $X$ is a quandle. We end in Section 5 with some open questions for future research.

*Email: Sam.Nelson@cmc.edu. Partially supported by Simons Foundation collaboration grant 316709

$\dagger$ Email: orrison@hmc.edu.

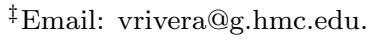




\section{Biquandles}

A biquandle is a set $X$ with two binary operations $\unrhd, \bar{\nabla}: X \times X \rightarrow X$ satisfying for all $x, y, z \in X$

(i) $x \unrhd x=x \bar{\triangleright} x$,

(ii) the maps $\alpha_{y}, \beta_{y}: X \rightarrow X$ and $S: X \times X \rightarrow X \times X$ defined by $\alpha_{y}(x)=x \bar{\triangleright} y, \beta_{y}(x)=x \unrhd y$ and $S(x, y)=(y \bar{\triangleright} x, x \unrhd y)$ are invertible, and

(iii) the exchange laws are satisfied:

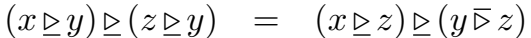

$$
\begin{aligned}
& (x \unrhd y) \bar{D}(z \unrhd y)=(x \bar{\nabla} z) \unrhd(y \bar{\nabla} z) \\
& (x \bar{\triangleright} y) \bar{\triangleright}(z \bar{\triangleright} y) \quad=\quad(x \bar{\triangleright} z) \bar{\triangleright}(y \unrhd z) .
\end{aligned}
$$

If $x \bar{\triangleright} y=x$ for all $x, y \in X$, we say $X$ is a quandle.

If $X$ and $Y$ are biquandles, then a biquandle homomorphism is a map $f: X \rightarrow Y$ such that for all $x, y \in X$, we have

$$
f(x \unrhd y)=f(x) \unrhd f(y) \quad \text { and } \quad f(x \bar{\nabla} y)=f(x) \triangleright f(y) .
$$

The biquandle axioms come from the Reidemeister moves where we interpret $x \unrhd y$ as $x$ crossing under $y$ and $y \bar{\triangleright} x$ as $y$ crossing over $x$ from left to right when the crossing has both strands oriented down as shown.
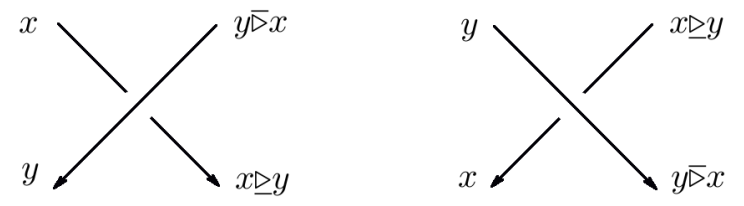

Remark 1. Note that there are four oriented Reidemeister I moves, four oriented Reidemeister II moves, and eight oriented Reidemeister III moves. In [16] several generating sets of oriented Reidemeister moves are identified; by Theorem 1.2 of [16], the set of moves including all four Type I moves, all four type II moves and the single type III move with all positive crossings is a generating set of oriented Reidemeister moves. In particular, for biquandles and the biquandle brackets in the next section, we will consider only these nine moves.

Remark 2. We are using the notation for biquandles from [6]; note that in the literature, particularly in older papers, it was more common to use the "downward" operations rather than our "sideways" operations. The newer notation is preferable for several reasons: the axioms are more symmetric and easier to remember, and the boundary map in biquandle homology is much simpler with this notation. See [6] for more details and further discussion.

Then the biquandle axioms are the conditions required for every valid biquandle coloring of the semiarcs in a knot diagram before a move to correspond to a unique valid biquandle coloring (i.e., coloring satisfying the condition pictured above at every crossing) of the diagram after the move. All four oriented type I moves require that $x \unrhd x=x \triangleright x$.
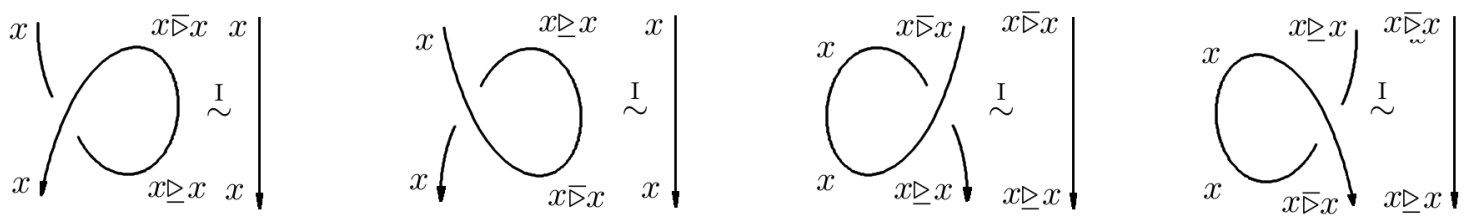
The direct type II moves, in which the strands are oriented in the same direction, require that $y \bar{\triangleright} x$ and $x \unrhd y$ are right-invertible.

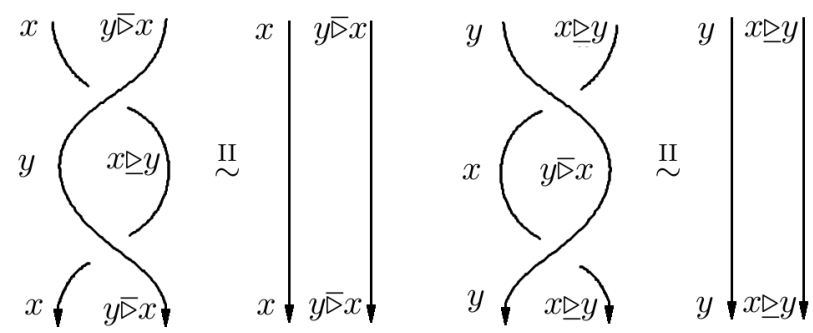

The reverse type II moves, in which the strands are oriented in opposite directions, require the map $(x, y) \mapsto$ $(y \bar{\triangleright} x, x \unrhd y)$ to be invertible.

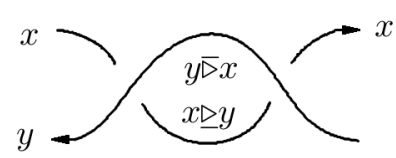

$\stackrel{\text { II }}{\sim}$

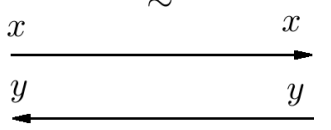

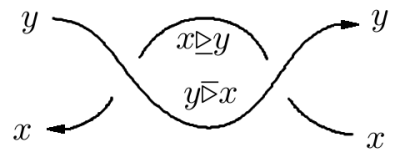

$\stackrel{\text { II }}{\sim}$

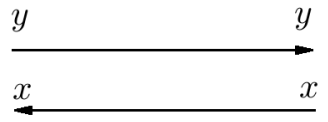

Finally, the exchange laws result from the Reidemeister III move.
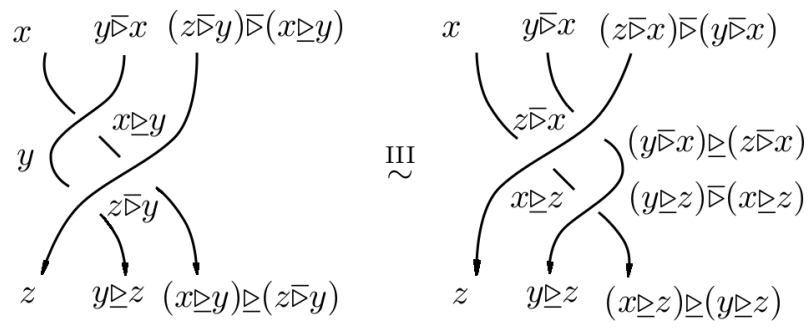

Example 1. Let $X$ be any set and $\sigma: X \rightarrow X$ any bijection. Then $X$ is a biquandle with operations

$$
x \unrhd y=x \triangleright y=\sigma(x)
$$

known as a constant action biquandle. If $\sigma$ is the identity, then $X$ is a trivial quandle.

Example 2. Let $\ddot{\Lambda}=\mathbb{Z}\left[t^{ \pm 1}, r^{ \pm 1}\right]$. Then any $\ddot{\Lambda}$-module $A$ is a biquandle, known as an Alexander biquandle, under the operations

$$
x \unrhd y=t x+\left(r^{-1}-t\right) y \quad \text { and } \quad x \bar{\triangleright} y=r^{-1} y .
$$

In particular, any commutative ring $A$ becomes an Alexander biquandle with a choice of invertible elements $t, r \in A$.

We can express the biquandle operations on a set $X=\left\{x_{1}, \ldots, x_{n}\right\}$ with operation tables for $\unrhd$ and $\bar{\nabla}$ expressed as an $n \times 2 n$ block matrix such that the entries in row $k$ columns $j$ and $n+j$ are the subscripts of $x_{k} \unrhd x_{j}$ and $x_{k} \bar{\triangleright} x_{j}$ respectively. 
Example 3. The Alexander biquandle structure on $\mathbb{Z}_{5}=\{1,2,3,4,5\}$ (where 5 represents the class of 0 so our block rows and columns are numbered 1 through 5) with $t=2$ and $r=4$ can be expressed as the block matrix

$$
\left[\begin{array}{lllll|lllll}
4 & 1 & 3 & 5 & 2 & 4 & 4 & 4 & 4 & 4 \\
1 & 3 & 5 & 2 & 4 & 3 & 3 & 3 & 3 & 3 \\
3 & 5 & 2 & 4 & 1 & 2 & 2 & 2 & 2 & 2 \\
5 & 2 & 4 & 1 & 3 & 1 & 1 & 1 & 1 & 1 \\
2 & 4 & 1 & 3 & 5 & 5 & 5 & 5 & 5 & 5
\end{array}\right]
$$

Example 4. Let $L$ be a tame oriented knot or link. The fundamental biquandle of $L$, denoted $\mathcal{B}(L)$, is the set of equivalence classes of biquandle words in a set of generators corresponding with the semiarcs in a diagram of $L$ under the equivalence relation generated by the crossing relations of $L$ and the biquandle axioms. For instance, the trefoil knot $3_{1}$

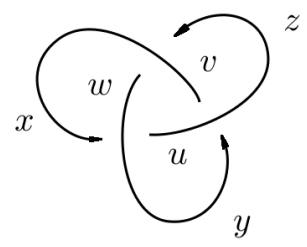

has the fundamental biquandle presentation

$$
\mathcal{B}\left(3_{1}\right)=\langle x, y, z, u, v, w \mid x \unrhd y=u, y \bar{\triangleright} x=w, y \unrhd z=v, z \bar{\triangleright} y=u, z \unrhd x=w, x \bar{\triangleright} z=v\rangle .
$$

Then for instance in $\mathcal{B}\left(3_{1}\right)$ we have

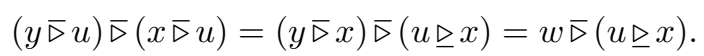

Different diagrams of the same knot or link yield different presentations which differ by Tietze moves and hence present the same biquandle.

Given a finite biquandle $X$ and a tame knot or link diagram $L$, a biquandle coloring of $L$ is an assignment of elements of $X$ to the semiarcs in $L$ such that the crossing relations
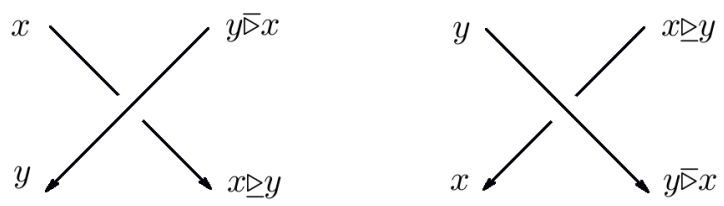

are satisfied at every crossing. Such an assignment determines and is determined by a biquandle homomorphism $f: \mathcal{B}(L) \rightarrow X$. In particular, the set of biquandle colorings of $L$ can be identified with the set $\operatorname{Hom}(\mathcal{B}(L), X)$ of biquandle homomorphisms from the fundamental biquandle of $L$ to $X$. If $L$ is tame, then $\mathcal{B}$ is finitely generated with $2 n$ generators where $n$ is the number of semiarcs in $L$; hence $|\operatorname{Hom}(\mathcal{B}(L), X)| \leq|X|^{2 n}$. We usually write $|\operatorname{Hom}(\mathcal{B}(L), X)|=\Phi_{X}^{\mathbb{Z}}(L) \in \mathbb{N} ;$ this cardinality is known as the biquandle counting invariant [2].

Example 5. The figure 8 knot $4_{1}$ below has only five valid biquandle coloring by the Alexander biquandle in example 3, as can be determined by row-reducing over $\mathbb{Z}_{5}$ the coefficient matrix of the system of crossing 
equations or by brute-force checking all possible colorings and counting those which satisfy the crossing relations. These colorings are pictured below:
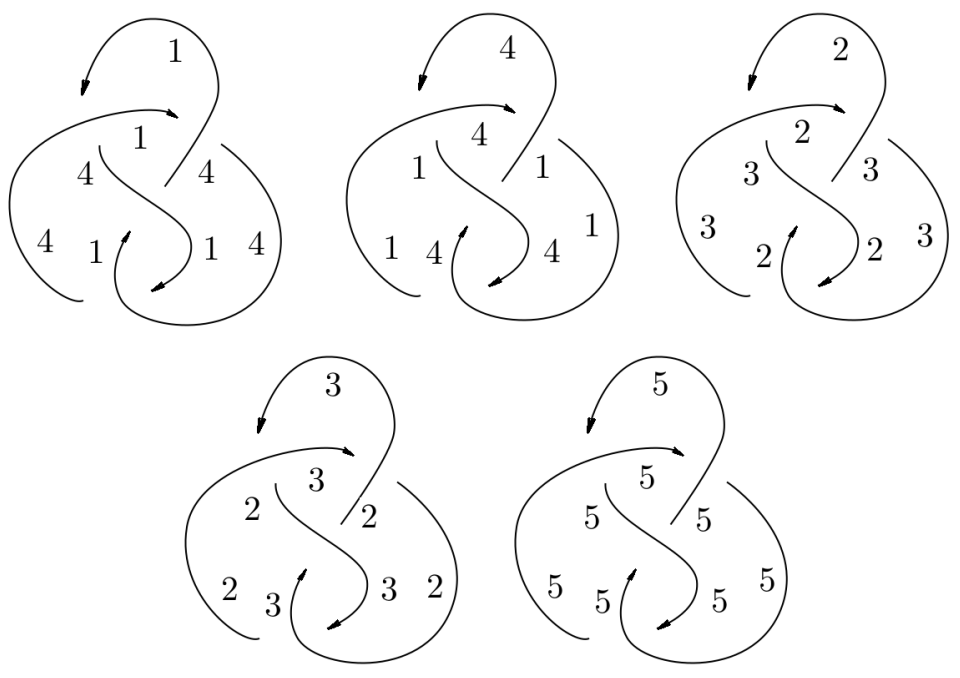

\section{Biquandle Brackets}

We would like to define a skein invariant (see [13] for instance) for biquandle-labeled link diagrams. Let $X$ be a finite biquandle, and let us fix a commutative ring with identity $R$ and denote the set of units of $R$ as $R^{\times}$. We would like to choose elements $A_{x, y}, B_{x, y}, w \in R^{\times}$and $\delta \in R$ such that the element of $R$ determined by the skein relations
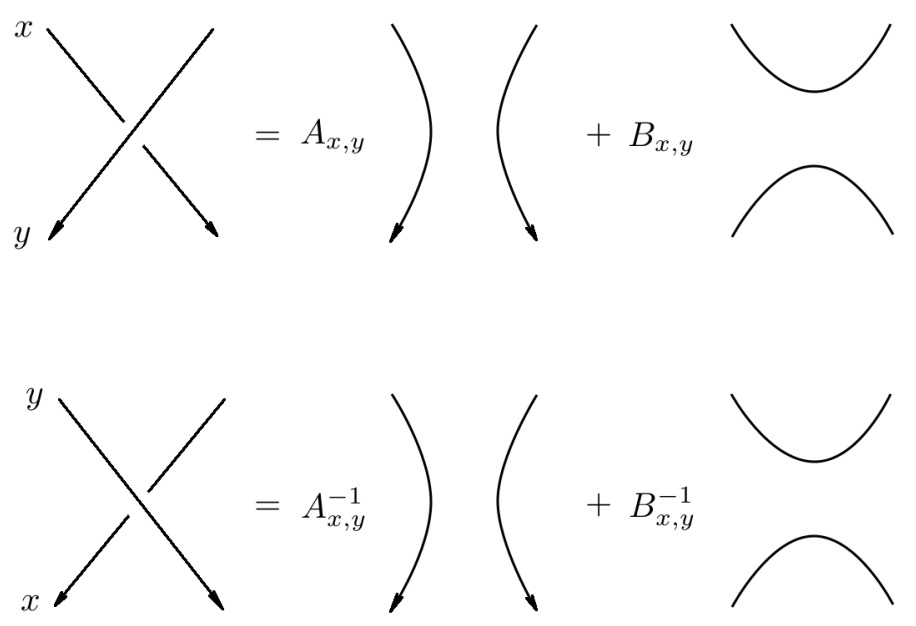

with $\delta$ the value of a simple closed curve and $w$ the value of a positive kink is an invariant of $X$-labeled Reidemeister moves. For a given choice of $X$ and $R$, we will denote such a collection of $A_{x, y}, B_{x, y}, w$ and $\delta$ by $\beta$.

More precisely, for each $X$-coloring $f$ of an oriented link diagram $D$ with $c$ crossings, we will find the collection of $2^{c}$ Kauffman states obtained by smoothing all the crossings as depicted above; to each such state will be associated a value in $R$ consisting of the product of the smoothing coefficients at crossings with 
$w^{n-p} \delta^{k}$ where $k$ is the number of circles in the Kauffman state, $n$ is the number of negative crossings and $p$ is the number of positive crossings in $D$. The sum of these contributions from each state will be denoted $\beta(f)$. The multiset of $\beta(f)$-values over the set of $X$-colorings will then be an invariant of oriented knots and links, which we will denote by $\Phi_{X}^{\beta, M}$; here the subscript $X$ indicates the coloring biquandle, the $\beta$ specifies the enhancement of the $X$-counting invariant, and the $M$ indicates the multiset version of the invariant.

Remark 3. It is standard practice for enhancements of counting invariants to be expressed in "polynomial form" by writing elements of the multiset as exponents of a formal variable $u$ with positive integer multiplicities as coefficients. We note that while strictly speaking this only defines a genuine (Laurent) polynomial in case $R=\mathbb{Z}$, this notation in common in the literature - it was introduced with quandle cocycle invariants in [4] and has been standard ever since, see [2, 3, 5, 6, 9, 10, 14, 15] for instance. The invariant written in this format contains the same information as the multiset version and has certain advantages; for instance, evaluation of $\Phi_{X}^{\beta}$ at $u=1$ (using the rule $1^{r}=1$ for all $r \in R$ ) yields the cardinality of the multiset version of the invariant, i.e. the $X$-counting invariant:

$$
\Phi_{X}^{\mathbb{Z}}(K)=\left.\Phi_{X}^{\beta}(K)\right|_{u=1} .
$$

Moreover, for certain brackets (as we will see later) using this format enables a factorization of the biquandle bracket polynomial as a product of a specialization of the Kauffman bracket polynomial with a biquandle cocycle polynomial. Additionally, the reader may find it easier to compare polynomials at a glance than to compare multisets. Of course, if preferred one can always use the multiset notation; we will generally use the polynomial form when $R=\mathbb{Z}$ or $\mathbb{Z}_{n}$ and the multiset form otherwise.

Let us address the obvious objection right away: smoothing a crossing in an $X$-labeled oriented link diagram does indeed result in diagrams without coherent biquandle colorings or even orientations. It follows that some modifications are needed if we wish to define the invariant recursively, as is often done with skein invariants; this will be a topic for another paper. However, we note that such a recursive definition is not necessary, since we can instead simply define the invariant via the state-sum formulation, i.e., define a function on oriented $X$-colored link diagrams by the summing the products of crossing coefficients times appropriate powers of $\delta$ and $w$ over the set of all completely smoothed states, with invariance following from the fact that this value is unchanged by $X$-colored Reidemeister moves. We can conceptualize this state-sum method as moving the biquandle colors off the crossings and into the coefficients for each state.

Recall that the set of four oriented Reidemeister I moves, four oriented Reidemeister II moves and the single Reidemeister III move with all positive crossings forms a generating set of oriented Reidemeister moves [16. Moreover, we have the following observation:

Observation 1. In a state sum defined as a sum over the set of states of the product of crossing weights times $\delta^{k}$ where $k$ is the number of circles in a state, local moves which preserve boundary connectivity, number of circles in each state and local crossing weight (i.e. coefficient product) do not change the state sum.

Thus, we can find the conditions a biquandle bracket $\beta$ must satisfy to define an invariant by identifying conditions such that the local crossing weights are preserved by the above-identified list of Reidemeister moves.

The first Reidemeister move comes in four oriented versions; the two positively oriented moves require that for all $x \in X$, we have $A_{x, x} \delta+B_{x, x}=w$, while the negatively oriented moves require $A_{x, x}^{-1} \delta+B_{x, x}^{-1}=w^{-1}$. In particular, we can think of writhe-reducing type I moves as factoring out powers of $w$ and writhe-increasing type I moves as factoring out powers of $w^{-1}$.

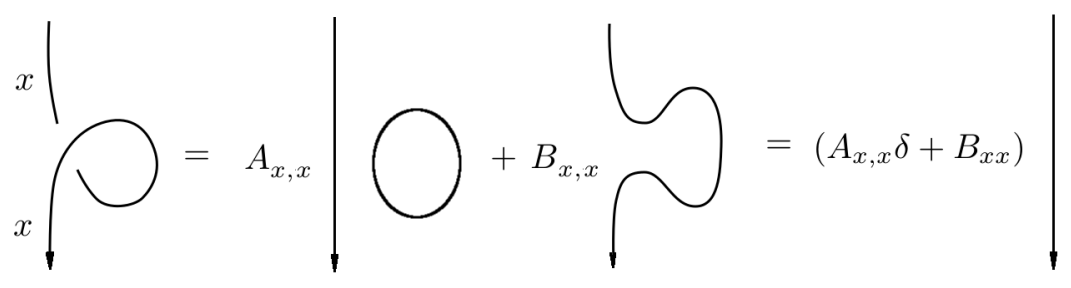


The direct type II moves require the oriented smoothing coefficients at positive and negative crossings to be multiplicative inverses, with the reverse II moves requiring the same of the unoriented smoothing coefficients; all four moves then require that $\delta=-A_{x, y} B_{x, y}^{-1}-A_{x, y}^{-1} B_{x, y}$.
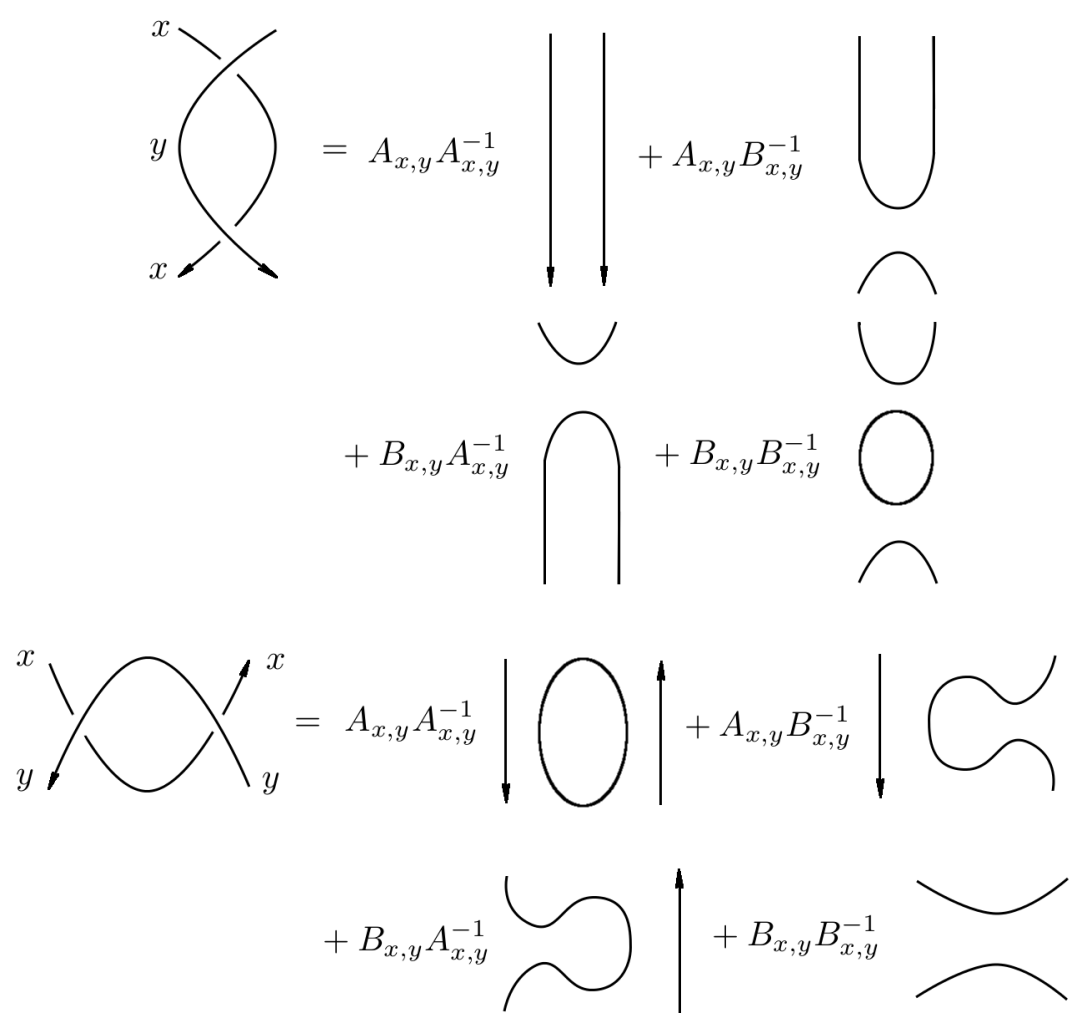

Comparing coefficients of the five crossingless diagrams

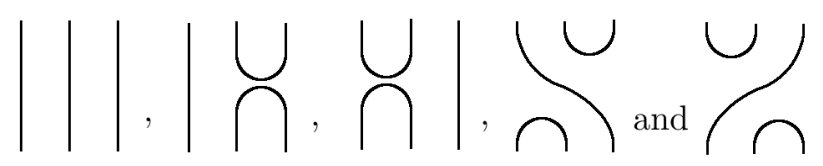


on both sides of the $X$-labeled Reidemeister III move, we have on the left side
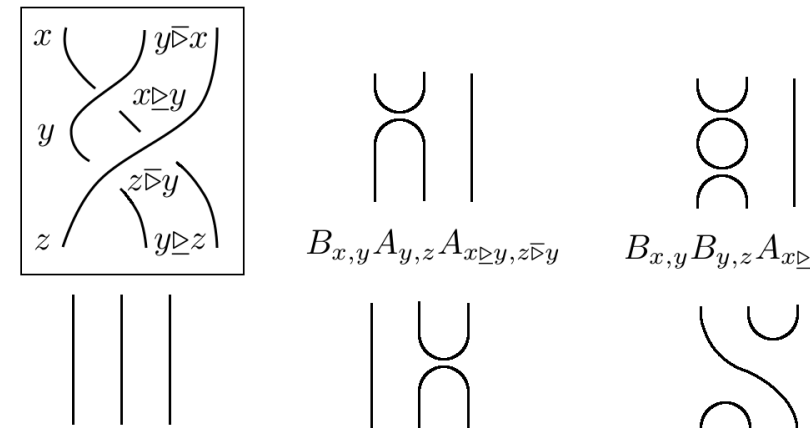

$B_{x, y} A_{y, z} A_{x \unrhd y, z \bar{\triangleright} y}$

$B_{x, y} B_{y, z} A_{x \unrhd y, z \bar{\nabla} y}$
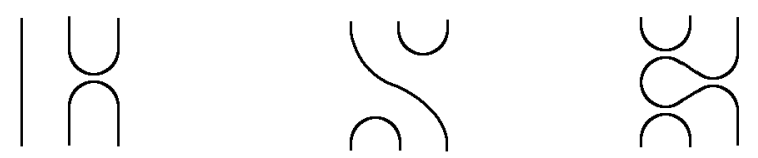

$A_{x, y} A_{y, z} A_{x \unrhd y, z \bar{\triangleright} y}$

$A_{x, y} A_{y, z} B_{x \unrhd y, z \triangleright y}$

$A_{x, y} B_{y, z} B_{x \unrhd y, z \triangleright \bar{y}}$

$B_{x, y} B_{y, z} B_{x \unrhd y, z \bar{\Sigma} y}$
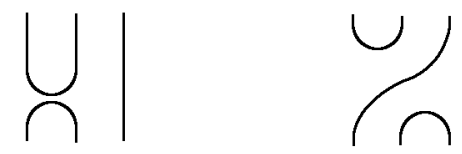

$A_{x, y} B_{y, z} A_{x \unrhd y, z \bar{\triangleright} y}$

$B_{x, y} A_{y, z} B_{x \unrhd y, z \triangleright y}$

and on the right side
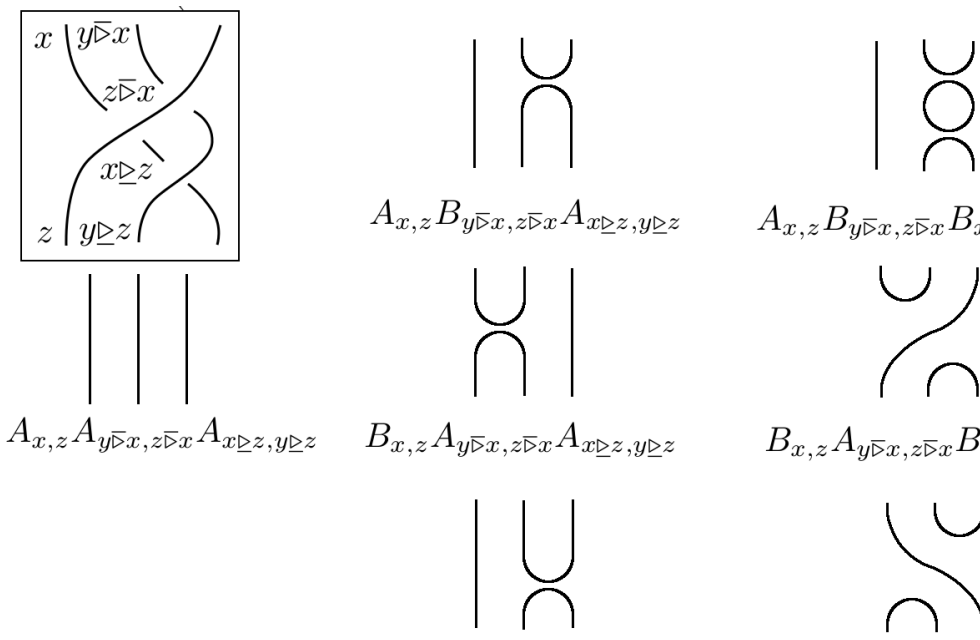

$A_{x, z} B_{y \bar{\triangleright} x, z \bar{\triangleright} x} B_{x \unrhd z, y \unrhd z}$
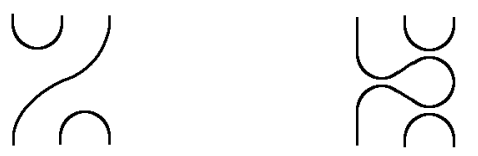

$B_{x, z} A_{y \bar{\triangleright} x, z \bar{\triangleright} x} B_{x \unrhd z, y \unrhd z}$

$B_{x, z} B_{y \bar{\triangleright} x, z \bar{\triangleright} x} B_{x \unrhd z, y \unrhd z}$

$A_{x, z} A_{y \bar{\triangleright} x, z \bar{\triangleright} x} B_{x \unrhd z, y \unrhd z}$

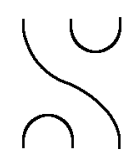

$B_{x, z} B_{y \varpi x, z \bar{\triangleright} x} A_{x \unrhd z, y \unrhd z}$

yielding the remaining conditions on $A_{x, y}$ and $B_{x, y}$. We thus obtain

Definition 1. Let $X$ be a finite biquandle and $R$ be a commutative ring with identity. A biquandle bracket on $X$ with values in $R$, also called an $X$-bracket, is a pair of maps $A, B: X \times X \rightarrow R^{\times}$and distinguished elements $\delta \in R$ and $w \in R^{\times}$satisfying

(i) for all $x \in X$,

$$
\delta A_{x, x}+B_{x, x}=w \quad \text { and } \quad \delta A_{x, x}^{-1}+B_{x, x}^{-1}=w^{-1}
$$

(ii) for all $x, y \in X$,

$$
\delta=-A_{x, y} B_{x, y}^{-1}-A_{x, y}^{-1} B_{x, y}
$$

and 
(iii) for all $x, y, z \in X$,

$$
\begin{aligned}
& A_{x, y} A_{y, z} A_{x \unrhd y, z \bar{\Sigma} y}=A_{x, z} A_{y \triangleright x, z \overline{ }} A_{x \unrhd z, y \unrhd z} \\
& A_{x, y} B_{y, z} B_{x \unrhd y, z \bar{\Sigma} y}=B_{x, z} B_{y \bar{\triangleright} x, z \bar{\Sigma} x} A_{x \unrhd z, y \unrhd z} \\
& B_{x, y} A_{y, z} B_{x \unrhd y, z \bar{y} y}=B_{x, z} A_{y \bar{\triangleright} x, z \bar{\Sigma}} B_{x \unrhd z, y \unrhd z} \\
& A_{x, y} A_{y, z} B_{x \unrhd y, z \bar{\triangleright} y}=A_{x, z} B_{y \bar{\triangleright} x, z \bar{\Sigma} x} A_{x \unrhd z, y \unrhd z}+A_{x, z} A_{y \bar{\triangleright} x, z \bar{\Sigma} x} B_{x \unrhd z, y \unrhd z} \\
& B_{x, y} A_{y, z} A_{x \unrhd y, z \bar{\triangleright} y}+A_{x, y} B_{y, z} A_{x \unrhd y, z \bar{\triangleright} y} \\
& +\delta A_{x, z} B_{y \bar{\Sigma} x, z \triangleright x} B_{x \unrhd z, y \unrhd z}+B_{x, z} B_{y \triangleright x, z \triangleright x} B_{x \unrhd z, y \unrhd z} \\
& +\delta B_{x, y} B_{y, z} A_{x \unrhd y, z \varpi y}+B_{x, y} B_{y, z} B_{x \unrhd y, z \triangleright y}=B_{x, z} A_{y \triangleright x, z \varpi x} A_{x \unrhd z, y \unrhd z}
\end{aligned}
$$

where $A(x, y)$ and $B(x, y)$ are denoted $A_{x, y}$ and $B_{x, y}$.

Given a finite biquandle $X=\left\{x_{1}, \ldots, x_{n}\right\}$, an $X$-bracket can be represented by a pair of $n \times n$ matrices $A, B$ with $A_{j, k}=A\left(x_{j}, x_{k}\right)$ and $B_{j, k}=B\left(x_{j}, x_{k}\right)$. We will usually write these as a single $n \times 2 n$ block matrix for convenience. Note that we can recover $\delta$ and $w$ from such a matrix, with

$$
\delta=-A_{1,1} B_{1,1}^{-1}-A_{1,1}^{-1} B_{1,1} \quad \text { and } \quad w=A_{1,1} \delta+B_{1,1} .
$$

Example 6. Let $X=\{1\}$ be the biquandle with one element. Then the matrix

$$
\left[A \mid A^{-1}\right]
$$

where $A \in \mathbb{Z}\left[A^{ \pm 1}\right]$ is an invertible variable defines a biquandle bracket with

$$
\delta=-A\left(A^{-1}\right)^{-1}-\left(A^{-1}\right) A^{-1}=-A^{2}-A^{-2} \quad \text { and } \quad w=A\left(-A^{2}-A^{-2}\right)+A^{-1}=-A^{3} .
$$

Indeed, this is the Kauffman bracket (see for example [13, 17]).

Example 7. Let $X$ be a finite biquandle, $R$ be a commutative ring, and $C: X \rightarrow R^{\times}$be a map where we write $C_{x}$ for $C(x)$. Then the maps $A, B: X \times X \rightarrow R^{\times}$defined by

$$
A_{x, y}=B_{x, y}=C_{x} C_{y}^{-1} C_{x \unrhd y}^{-1} C_{y \overline{ }}
$$

for all $x, y \in X$ define a biquandle bracket with $\delta=-2$ and $w=-1$. To see this, we note that if $A_{x, y}=B_{x, y}$, we necessarily have $\delta=-2$, and biquandle bracket axiom (iii)'s five equations all reduce to the first equation, namely

$$
A_{x, y} A_{y, z} A_{x \unrhd y, z \bar{\triangleright} y}=A_{x, z} A_{y \bar{\triangleright} x, z \bar{\triangleright} x} A_{x \unrhd z, y \unrhd z} .
$$

Then

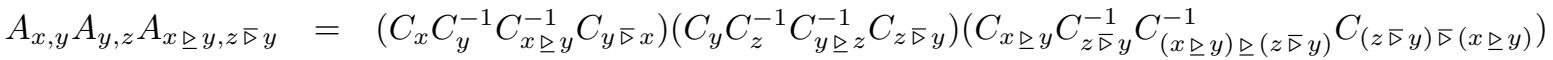

$$
\begin{aligned}
& =C_{x} C_{y \triangleright x} C_{z}^{-1} C_{y \unrhd z}^{-1} C_{(x \unrhd y) \unrhd(z \bar{\triangleright} y)}^{-1} C_{(z \triangleright y) \triangleright(x \unrhd y)}
\end{aligned}
$$

while

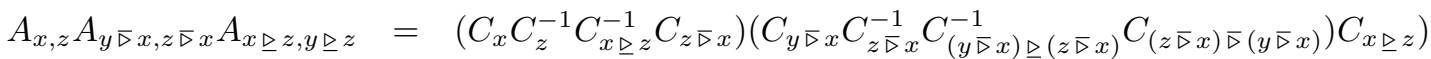

$$
\begin{aligned}
& \times\left(C_{y \unrhd z}^{-1} C_{(x \unrhd z) \unrhd(y \unrhd z)}^{-1} C_{(y \unrhd z) \triangleright(x \unrhd z)}\right) \\
& =C_{x} C_{z}^{-1} C_{y \triangleright x} C_{(z \triangleright x) \triangleright(y \triangleright x)} C_{y \unrhd z}^{-1} C_{(x \unrhd z) \unrhd(y \unrhd z)}^{-1}
\end{aligned}
$$

which are equal by the exchange laws. As we will later see, this type of biquandle bracket is actually a biquandle 2-coboundary; in particular, two biquandle brackets which differ by a coboundary are "cohomologous" and define the same invariant.

We now introduce the first of our new invariants. 
Definition 2. Let $L$ be an oriented knot or link diagram with $n$ crossings with generators $x_{1}, \ldots, x_{2 n}$ for the fundamental biquandle $\mathcal{B}(L)$ associated to the semiarcs. There are $2^{n}$ states corresponding to choices of oriented or unoriented smoothing for each crossing, each of which has an associated product of $n$ factors of $A_{x, y}^{ \pm 1}$ or $B_{x, y}^{ \pm 1}$ times $\delta^{k}$ where $k$ is the number of components of the state. The sum of these contributions times the writhe correction factor, $w^{n-p}$, is the fundamental biquandle bracket value for $L$.

Example 8. The Hopf link $L 2 a 1$ below has four smoothed states with coefficients as listed.
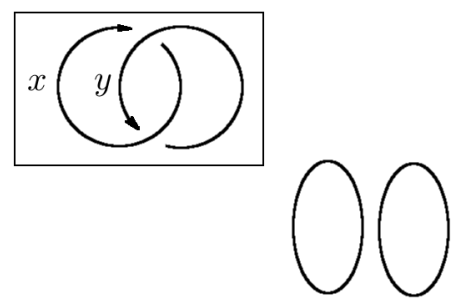

$A_{x, y} A_{y, x}$
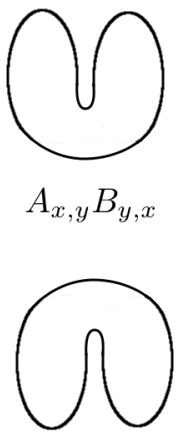

$B_{x, y} A_{y, x}$

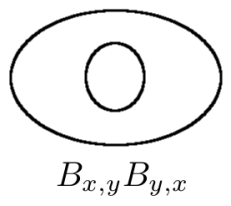

Then the Hopf link has fundamental biquandle bracket value

$$
\phi=w^{-2}\left(A_{x, y} A_{y, x} \delta^{2}+B_{x, y} A_{y, x} \delta+A_{x, y} B_{y, x} \delta+B_{x, y} B_{y, x} \delta^{2}\right)
$$

where $x, y$ are generators of the fundamental biquandle $\mathcal{B}(L 2 a 1)=\langle x, y \mid x \unrhd y=x \bar{\triangleright} y, y \unrhd x=y \bar{\triangleright} x\rangle$.

The fundamental biquandle bracket treats every knot or link as colored by elements of its fundamental biquandle. This fundamental biquandle bracket may be a complete invariant of knots since it includes the fundamental biquandle and hence the fundamental quandle, already known to be a complete invariant for virtual knots up to a type of reflection [11, and our later examples demonstrate that the fundamental biquandle bracket can detect mirror images. However, comparing fundamental biquandle bracket values for different knots and links is not straightforward since any two such links are being colored by generally different biquandles.

To get a more immediately useful invariant, let $X$ be a finite biquandle. For any $X$-bracket $\beta$ over $R$, evaluating the fundamental biquandle bracket value of an $X$-coloring $f$ of an oriented link diagram $L$ yields an element of $R$ which is unchanged by $X$-colored Reidemeister moves on $L$; let us denote this value by $\beta(f)$.

Definition 3. Let $X$ be a finite biquandle, $L$ an oriented link and $\beta$ a biquandle bracket. Then the biquandle bracket multiset invariant of $L$ is the multiset of $\beta$-values over the set of $X$-labelings of $L$,

$$
\Phi_{X}^{\beta, M}(L)=\{\beta(f) \mid f \in \operatorname{Hom}(\mathcal{B}(L), X)\}
$$

and the biquandle bracket polynomial invariant of $L$ is

$$
\Phi_{X}^{\beta}(L)=\sum_{f \in \operatorname{Hom}(\mathcal{B}(L), X)} u^{\beta(f)} .
$$

Remark 4. Again, note that in this "polynomial" form, the coefficients are integers, $u$ is a formal variable and the exponents of $u$ are elements of $R$.

Proposition 1. Let $X$ be a finite biquandle and let $\beta$ and $\beta^{\prime}$ be $X$-brackets over $R$ defined by maps $A, B$ : $X \times X \rightarrow R^{\times}$and $A^{\prime}, B^{\prime}: X \times X \rightarrow R^{\times}$respectively. If there is an invertible scalar $\alpha \in R^{\times}$such that for all $x, y \in X$ we have

$$
A_{x, y}=\alpha A_{x, y}^{\prime} \quad \text { and } \quad B_{x, y}=\alpha B_{x, y}^{\prime}
$$

then the link invariants defined by $\beta$ and $\beta^{\prime}$ are equal. 
Proof. First, we note that

$$
\delta^{\prime}=-A_{x, y}^{\prime} B_{x, y}^{\prime-1}-A_{x, y}^{\prime-1} B_{x, y}^{\prime}=-\left(\alpha A_{x, y}\right)\left(\alpha B_{x, y}\right)^{-1}-\left(\alpha A_{x, y}^{-1}\right) \alpha B_{x, y}=-A_{x, y} B_{x, y}^{-1}-A_{x, y}^{-1} B_{x, y}=\delta
$$

and

$$
w^{\prime}=A_{x, x}^{\prime} \delta+B_{x, x}^{\prime}=\alpha A_{x, x} \delta+\alpha B_{x, x}=\alpha w .
$$

Then for any link diagram $L$ with $j$ positive crossings and $k$ negative crossings, the state sum with $\beta^{\prime}$ equals that with $\beta$ multiplied by $\alpha^{j-k}$ at every crossing. Then the contribution $\beta^{\prime}(f)$ equals $\beta(f)$ multiplied by $\alpha^{k-k}$, then multiplied by $\alpha^{k-j}$ in the writhe-correction factor $\left(w^{\prime}\right)^{k-j}$; hence, the powers of $\alpha$ cancel and we have $\beta(f)=\beta^{\prime}(f)$, whence $\Phi_{x}^{\beta}(L)=\Phi_{x}^{\beta^{\prime}}(L)$ for all classical and virtual knots and links $L$.

Example 9. The simplest non-trivial biquandle is the constant action biquandle on $X=\{1,2\}$ with operation matrix

$$
\left[\begin{array}{ll|ll}
2 & 2 & 2 & 2 \\
1 & 1 & 1 & 1
\end{array}\right]
$$

The counting invariant $\Phi_{X}^{\mathbb{Z}}(L)$ with respect to this biquandle is 0 if $L$ is a virtual link with any component containing an odd number of crossing points and is $2^{c}$ where $c$ is the number of components of $L$ otherwise. Our python computations reveal biquandle bracket structures on $X$ with coefficients in $\mathbb{Z}_{5}$ including

$$
\left[\begin{array}{ll|ll}
1 & 3 & 4 & 2 \\
4 & 1 & 1 & 4
\end{array}\right]
$$

The Hopf link has four $X$-labelings and fundamental biquandle bracket value

$$
\phi=A_{x, y} A_{y, x} \delta^{2}+B_{x, y} A_{y, x} \delta+A_{x, y} B_{y, x} \delta+B_{x, y} B_{y, x} \delta^{2} .
$$

Then we have $\delta=2, w=1$ and

\begin{tabular}{cc|c}
$x$ & $y$ & $\phi$ \\
\hline 1 & 1 & $1(1)\left(2^{2}\right)+1(4)(2)+4(1)(2)+4(4)\left(2^{2}\right)=4+3+3+4=4$ \\
1 & 2 & $3(4)\left(2^{2}\right)+2(4)(2)+3(1)(2)+2(1)\left(2^{2}\right)=3+1+1+3=3$ \\
2 & 1 & $4(3)\left(2^{2}\right)+1(3)(2)+4(2)(2)+1(2)\left(2^{2}\right)=3+1+1+3=3$ \\
2 & 2 & $1(1)\left(2^{2}\right)+1(4)(2)+4(1)(2)+4(4)\left(2^{2}\right)=4+3+3+4=4$
\end{tabular}

or in more pictorial form,

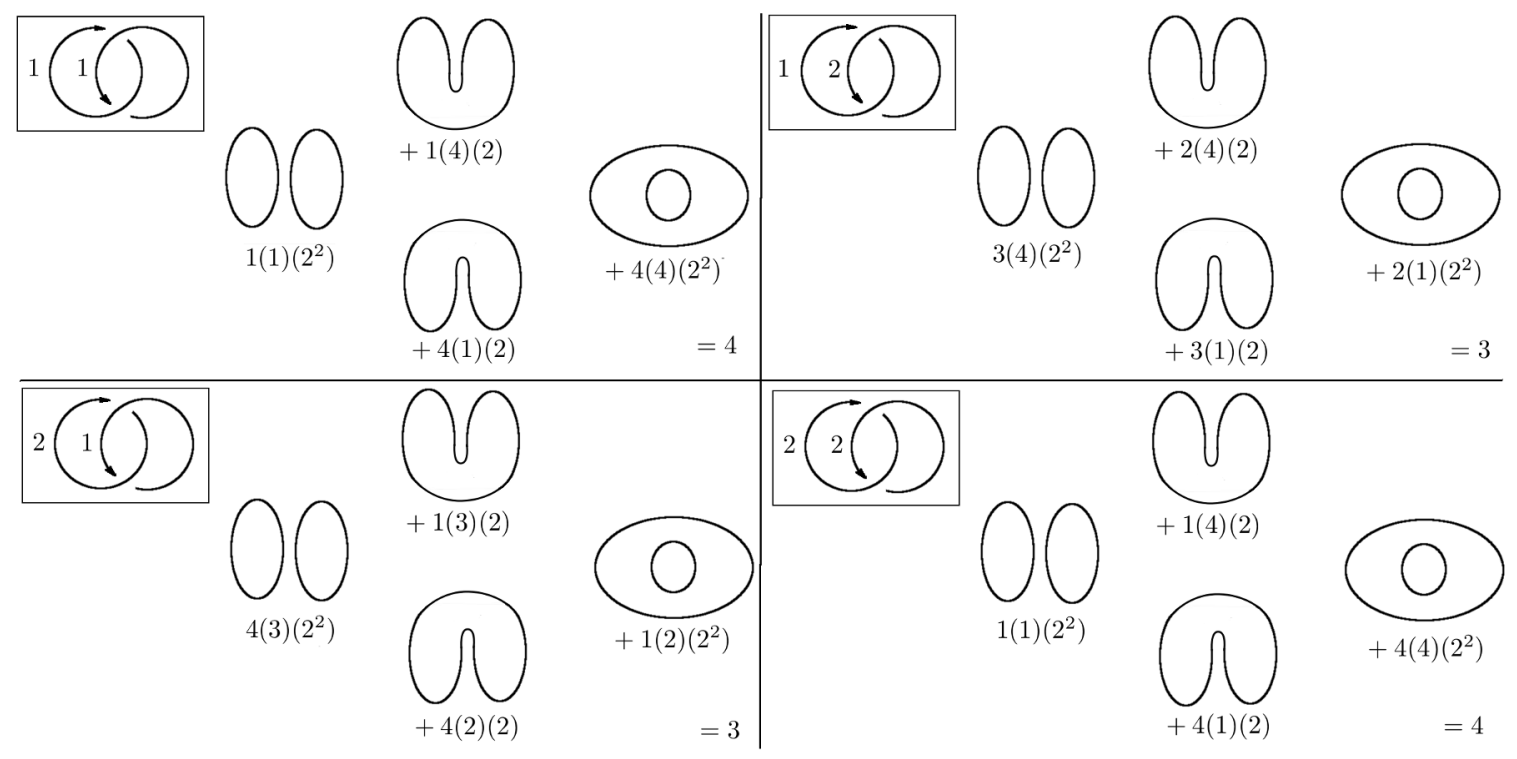


Then the Hopf link has biquandle bracket invariant

$$
\Phi_{X}^{\beta, M}(L)=\{3,3,4,4\}
$$

or in "polynomial" form

$$
\Phi_{X}^{\beta}(L)=2 u^{3}+2 u^{4}
$$

while the unlink of two components $U_{2}$ has invariant value

$$
\Phi_{X}^{\beta}\left(U_{2}\right)=4 u^{4} .
$$

Example 10. Let $X$ be any finite biquandle and $R$ be any commutative ring with identity. For any invertible element $t \in R$, the maps $A_{x, y}=t, B_{x, y}=t^{-1}$ with $\delta=-t^{-2}-t^{2}$ and $w=t^{3}$ define a biquandle bracket $\beta_{t}$ called a constant biquandle bracket. Since in this case the skein coefficients do not depend on the biquandle colors, each $X$-coloring gets the same state-sum value, namely $\Phi_{X}^{\mathbb{Z}}(L) u^{K_{L}(t)}$ where $K_{L}(t)$ is the Kauffman bracket polynomial of $L$ evaluated at $t$; hence, for any link $L$, the biquandle bracket invariant with respect to $\beta_{t}$ is $\Phi_{X}^{\beta}(L)=\Phi_{X}^{\mathbb{Z}}(L) u^{K_{L}(t)}$.

For example, if $R=\mathbb{Z}_{7}$ and $t=2$, we have $2^{-1}=4, \delta=-4^{2}-2^{2}=-16-4=-20=1$ and $w=t^{3}=2^{3}=8=1$; then in the Hopf link example above we have

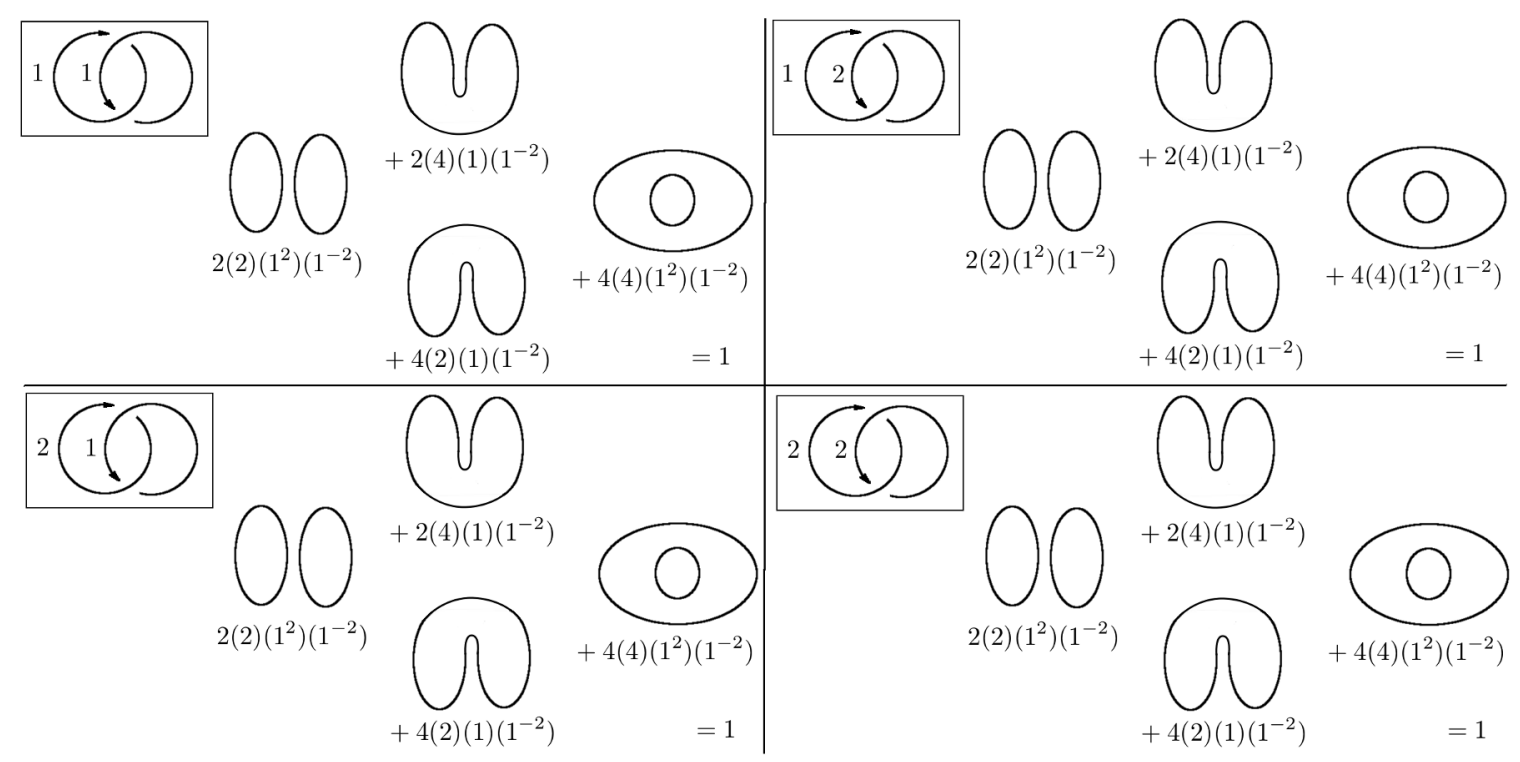

so we have

$$
\Phi_{X}^{\beta}(L)=4 u=\Phi_{X}^{\mathbb{Z}}(L) u^{K_{L}(t)}
$$

since we have $\Phi_{X}^{\mathbb{Z}}(L)=4$ and $K_{L}(2)=1+t^{-4}+t^{-8}+\left.t^{-12}\right|_{t=2}=1+4+2+1=1$.

Example 11. More generally, an $X$-bracket in which for all $x, y \in X$ we have $A_{x, y}=A$ and $B_{x, y}=B$ defines a skein invariant which does not use the biquandle colors; the biquandle bracket invariant will then be $\Phi_{X}^{\mathbb{Z}}(K)$ copies of the skein invariant thus defined. For instance, if $A+A^{-1}=B+B^{-1}$, we have a biquandle bracket $\beta$ satisfying $\Phi_{X}^{\beta_{A, B}}(L)=\Phi_{X}^{\mathbb{Z}}(L) u^{K_{L}\left(-A^{2} B^{-1}, A+A^{-1}\right)}$ where $K(a, z)$ is the Kauffman 2-variable polynomial, as one can easily see by comparing the biquandle bracket skein relation with the usual Kauffman 2-variable skein relation. Similarly, a biquandle bracket $\beta$ with $A_{x, y}=\alpha A$ and $B_{x, y}=B$ with $\alpha^{-1} B+\alpha B^{-1}=0$ yields $\Phi_{X}^{\beta_{a, z}}(L)=\Phi_{X}^{\mathbb{Z}}(L) u^{H_{L}\left(\alpha, \alpha^{-1} A+\alpha A^{-1}\right)}$ where $H(a, z)$ is the HOMFLY-PT polynomial [13]. 
Example 12. Let $X$ be a finite biquandle, $G$ an abelian group, and $\psi \in H^{2}(X ; G)$ an element of the second cohomology of $X$ with $G$ coefficients, i.e., a function $\psi: X \times X \rightarrow G$ satisfying for all $x, y, z \in X$

$$
\psi(x, y) \psi(y, z) \psi(x \bar{\triangleright} y, z \unrhd y)=\psi(x, z) \psi(y \bar{\triangleright} x, z \bar{\triangleright} x) \psi(x \unrhd z, y \unrhd z)
$$

and $\psi(x, x)=1$ (see [5] for instance). Then setting $A_{x, y}=B_{x, y}=\psi(x, y)$ defines a biquandle bracket with $R=\mathbb{Z}[G]$. Indeed, every biquandle bracket with $A_{x, y}=B_{x, y}$ for all $x, y \in X$ arises in this way, since the biquandle bracket conditions with $A_{x, y}=B_{x_{y}}$ reduce to $\delta=-2, w=-1$ and the 2-cocycle condition

$$
A_{x, y} A_{y, z} A_{x \unrhd y, z \bar{\nabla} y}=A_{x, z} A_{y \triangleright x, z \bar{\triangleright} x} A_{x \unrhd z, y \unrhd z} .
$$

The biquandle bracket invariant in this case satisfies

$$
\Phi_{X}^{\beta}(L)=\Phi_{X}^{\psi}(L) K_{L}(1)
$$

where $K_{L}(1)$ is the Kauffman bracket polynomial of $L$ evaluated at $A=1$.

Proposition 2. Let $X$ be a finite biquandle, $R$ be a commutative ring and $C: X \rightarrow R^{\times}$be a map as in example 7 and let $\gamma: X \times X \rightarrow \mathbb{R}^{\times}$be the biquandle bracket defined by setting both $A$ and $B$ equal to

$$
\gamma(x, y)=C(x) C(y)^{-1} C(x \unrhd y)^{-1} C(y \bar{\triangleright} x) .
$$

Then for any biquandle bracket $\beta$ defined by $A, B: X \times X \rightarrow R^{\times}$, the maps

$$
A_{x, y}^{\prime}=A_{x, y} \gamma(x, y) \quad \text { and } \quad B_{x, y}^{\prime}=B_{x, y} \gamma(x, y)
$$

define a biquandle bracket $\gamma \beta$ with $\delta=-A_{x, y} B_{x, y}^{-1}-A_{x, y}^{-1} B_{x, y}$ and we have $\Phi_{X}^{\beta}=\Phi_{X}^{\gamma \beta}$.

Proof. In $\gamma \beta$, the invertible quantity

$$
C(x) C(y \bar{\triangleright} x) C(z)^{-1} C(y \unrhd z)^{-1} C((x \unrhd y) \unrhd(z \bar{\triangleright} y))^{-1} C((z \bar{\triangleright} y) \bar{\triangleright}(x \unrhd y))
$$

factors out of each term on both sides of the equations in biquandle bracket axiom (iii), so $\gamma \beta$ is a biquandle bracket provided $\beta$ is.

To see that $\beta$ and $\gamma \beta$ define the same invariant, note that we can picture $\gamma \beta$ as including factors of $C(x)$, $C(y \triangleright x), C(y)^{-1}, C(x \unrhd y)^{-1}$ and on the initial and terminal ends of the semiarc respectively along with the $A_{x, y}$ and $B_{x, y}$ coefficients as shown.
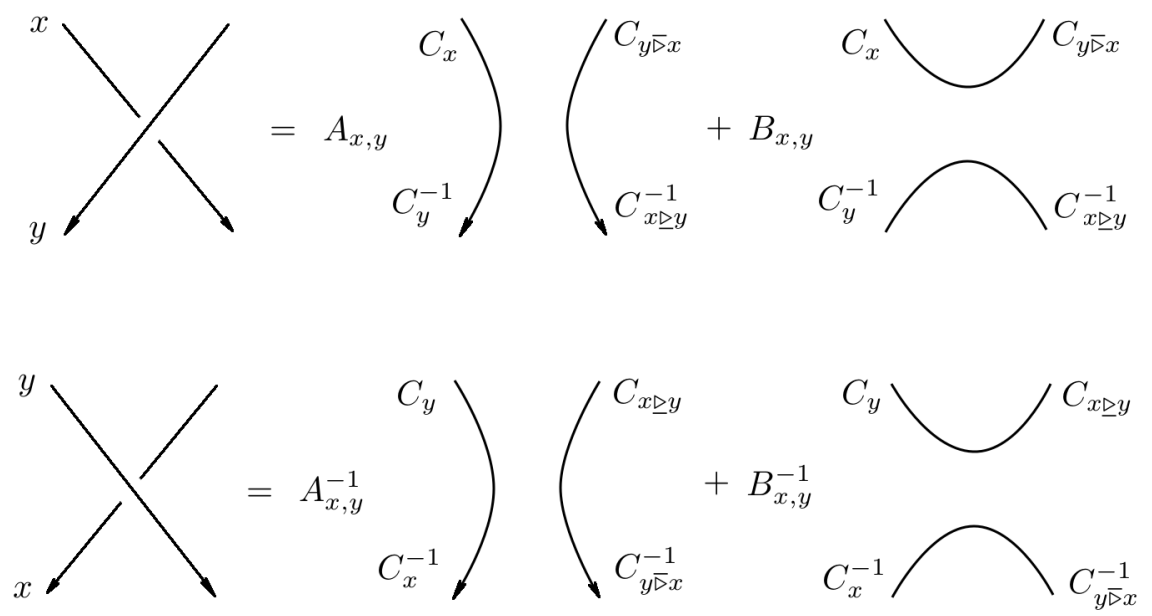

Then we observe that over any complete link diagram, the $C$ factors match up in canceling pairs along each semiarc, so the value of each state of an $X$-colored link in $\Phi_{X}^{C \beta}$ is the same as in $\Phi_{X}^{\beta}$. 
For biquandle brackets $\beta$ representing biquandle 2-cocycles, $\gamma$ is a coboundary and $\beta$ and $\gamma \beta$ are cohomologous; however, Proposition 2 holds even for biquandle brackets $\beta$ not representing cocycles. Thus, it is tempting to define $\beta$ and $\gamma \beta$ to be "cohomologous" regardless of whether $\beta$ is a cocycle; however, we will settle for the following:

Definition 4. Two $X$-brackets $\beta$ and $\beta^{\prime}$ over $R$ are $C$-equivalent if there is a map $C: X \rightarrow R^{\times}$such that for all $x, y \in X$, we have

$$
\begin{aligned}
& A_{x, y}^{\prime}=A_{x, y} C(x) C(y)^{-1} C(x \unrhd y)^{-1} C(y \bar{\triangleright} x) \text { and } \\
& B_{x, y}^{\prime}=B_{x, y} C(x) C(y)^{-1} C(x \unrhd y)^{-1} C(y \bar{\triangleright} x) .
\end{aligned}
$$

Corollary 3. C-equivalent $X$-brackets define the same invariant $\Phi_{X}^{\beta}$.

In [14, quantum enhancements of the counting invariant with respect to involutory biquandles $X$ were defined as functors from the category of $X$-labeled unoriented tangles to an $R$-module category. Biquandle brackets provide examples of quantum enhancements as defined in [14 in the following way: Given a biquandle bracket $\beta$, define

$$
I=\left[\begin{array}{ll}
1 & 0 \\
0 & 1
\end{array}\right], \quad N=\left[\begin{array}{llll}
0 & A_{11} & -B_{11} & 0
\end{array}\right] \quad \text { and } \quad U=\left[\begin{array}{c}
0 \\
-B_{11}^{-1} \\
A_{11}^{-1} \\
0
\end{array}\right] .
$$

Then the biquandle bracket skein relation yields $X$-labeled $R$-matrices $X_{x, y}^{ \pm 1}$ :

$$
\begin{aligned}
X_{x, y} & =A_{x, y}(I \otimes I)+B_{x, y}(U N) \\
& =\left[\begin{array}{cccc}
A_{x, y} & 0 & 0 & 0 \\
0 & 0 & B_{x, y} & 0 \\
0 & B_{x, y} & A_{x, y}-A_{x, y}^{-1} B_{x, y}^{2} & 0 \\
0 & 0 & 0 & A_{x, y}
\end{array}\right] .
\end{aligned}
$$

See [17] for more.

Example 13. The biquandle bracket in example 9 corresponds to quantum weight over $\mathbb{Z}_{5}$ given by

$$
\begin{gathered}
I=\left[\begin{array}{ll}
1 & 0 \\
0 & 1
\end{array}\right], \quad U=\left[\begin{array}{llll}
0 & 1 & 1 & 0
\end{array}\right], \quad N=\left[\begin{array}{l}
0 \\
1 \\
1 \\
0
\end{array}\right], \\
X_{1,1}=\left[\begin{array}{llll}
1 & 0 & 0 & 0 \\
0 & 0 & 4 & 0 \\
0 & 4 & 0 & 0 \\
0 & 0 & 0 & 1
\end{array}\right], \quad X_{1,2}=\left[\begin{array}{llll}
3 & 0 & 0 & 0 \\
0 & 0 & 2 & 0 \\
0 & 2 & 1 & 0 \\
0 & 0 & 0 & 3
\end{array}\right], \\
X_{2,1}=\left[\begin{array}{llll}
4 & 0 & 0 & 0 \\
0 & 0 & 1 & 0 \\
0 & 1 & 0 & 0 \\
0 & 0 & 0 & 4
\end{array}\right], \quad \text { and } X_{2,2}=\left[\begin{array}{llll}
1 & 0 & 0 & 0 \\
0 & 0 & 4 & 0 \\
0 & 4 & 0 & 0 \\
0 & 0 & 0 & 1
\end{array}\right] .
\end{gathered}
$$

In particular, this quantum enhancement is an example of a strongly heterogeneous quantum weight as defined in the questions in [14, since $X_{1,2}$ is not a classical $R$-matrix. 
Example 14. Let $X$ be the biquandle defined by the operation matrix

$$
\left[\begin{array}{ll|ll}
2 & 2 & 2 & 2 \\
1 & 1 & 1 & 1
\end{array}\right]
$$

and let $R=\mathbb{F}_{8}=\mathbb{Z}_{2}[t] /\left(1+t+t^{3}\right)$ be the Galois field of eight elements. That is, $R$ is the ring of polynomials in one variable with $\mathbb{Z}_{2}$ coefficients with the rule that $t^{3}=1+t$. Then our python computations reveal that

$$
\left[\begin{array}{cc|cc}
1 & 1+t & t & t+t^{2} \\
1+t^{2} & 1 & 1 & t
\end{array}\right]
$$

defines a biquandle bracket. We can describe this one without explicitly referencing biquandles in the following way: Given any oriented link $L$ of $c$ components, find the the $2^{c}$ ways to color the semiarcs of $L$ alternately solid and dotted going around each component (or for virtual links lacking such a coloring, set the invariant value to zero). Then for each such coloring, expand using the following skein relations 1
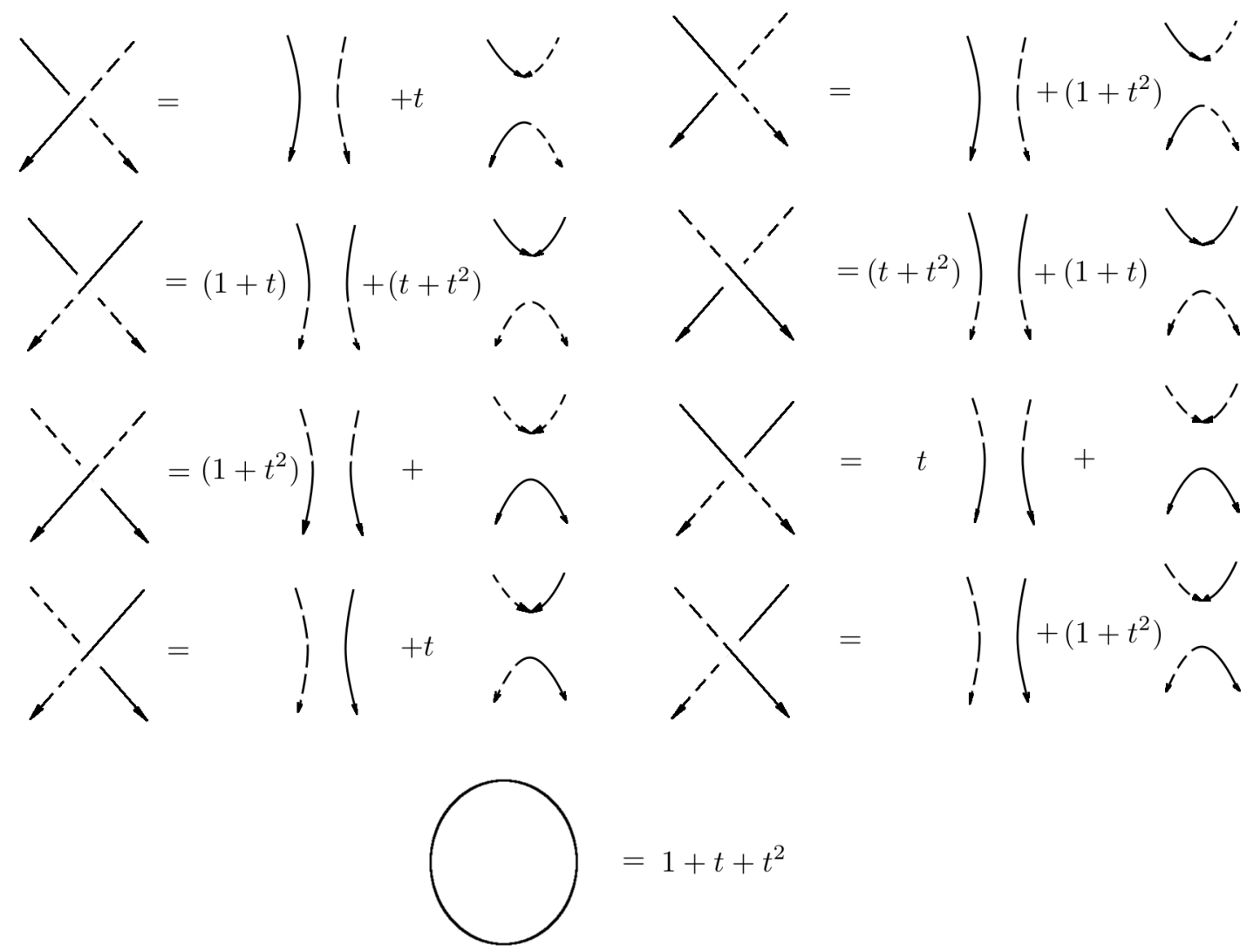

Note that while fully resolved closed curves have some solid and some dashed sections and lack globally consistent orientations, every closed curve evaluates simply to $1+t+t^{2}$. Finally, multiply by the writhe normalization factor $t^{p}\left(1+t^{2}\right)^{n}$ where $p$ and $n$ are the numbers of positive and negative crossings respectively and collect these values into a multiset over the set of all colorings of $L$.

We computed this invariant for all prime classical knots with up to eight crossings and all prime classical links with up to seven crossings as found in the tables at the knot atlas [1]. The results are collected in the

\footnotetext{
${ }^{1}$ Thanks to Zhiqing Yang for catching a misprint in an earlier version of this table.
} 
tables below. We list the multiset version of the invariant for ease of reading since this ring is not $\mathbb{Z}$ or $\mathbb{Z}_{n}$. We start with the prime classical knots:

\begin{tabular}{r|l}
$\Phi_{X}^{\beta}(K)$ & $K$ \\
$\{2 \times 1\}$ & $5_{2}, 7_{5}, 8_{10}, 8_{11}, 8_{13}, 8_{17}$ \\
$\{2 \times t\}$ & $3_{1}, 6_{2}, 8_{9}$ \\
$\{2 \times 1+t\}$ & $4_{1}, 7_{1}, 7_{4}, 8_{5}, 8_{14}$ \\
$\left\{2 \times t^{2}\right\}$ & $6_{1}, 6_{3}, 7_{2}, 7_{3}, 8_{7}, 8_{21}$ \\
$\left\{2 \times 1+t^{2}\right\}$ & $7_{7}, 8_{2}, 8_{3}, 8_{4}, 8_{8}, 8_{19}, 8_{20}$ \\
$\left\{2 \times t+t^{2}\right\}$ & $8_{1}, 8_{6}, 8_{12}, 8_{16}, 8_{18}$ \\
$\left\{2 \times 1+t+t^{2}\right\}$ & Unknot, $5_{1}, 7_{6}, 8_{15}$
\end{tabular}

For prime classical links with up to seven crossings, we have

\begin{tabular}{r|l}
$\Phi_{X}^{\beta}(L)$ & $L$ \\
\hline$\{2 \times 1,2 \times t\}$ & $L 6 a 2$ \\
$\left\{2 \times t, 2 \times 1+t^{2}\right\}$ & $L 7 a 6$ \\
$\left\{2 \times 1,2 \times t+t^{2}\right\}$ & $L 6 a 1$ \\
$\left\{2 \times 1,2 \times 1+t+t^{2}\right\}$ & $L 7 a 5$ \\
$\left\{2 \times t^{2}, 2 \times 1+t+t^{2}\right\}$ & $L 7 a 2, L 7 n 1$ \\
$\left\{2 \times 1+t^{2}, 2 \times t+t^{2}\right\}$ & $L 2 a 1$ \\
$\left\{2 \times 1+t^{2}, 2 \times 1+t\right\}$ & $L 4 a 1$ \\
$\left\{2 \times t+t^{2}, 2 \times 1+t+t^{2}\right\}$ & $L 6 a 3$ \\
$\left\{4 \times t^{2}\right\}$ & $L 5 a 1$ \\
$\left\{4 \times t+t^{2}\right\}$ & $L 7 a 1, L 7 a 3, L 7 a 4$ \\
$\left\{2 \times 1,6 \times t+t^{2}\right\}$ & $L 6 a 4, L 6 n 1$ \\
$\left\{2 \times t^{2}, 6 \times 1+t^{2}\right\}$ & $L 6 a 5$ \\
$\left\{2 \times t, 6 \times t+t^{2}\right\}$ & $L 7 a 7$.
\end{tabular}

We note that:

- $\Phi_{X}^{\beta}$ distinguishes the right- and left-hand trefoils, with invariant values of $\{2 \times t\}$ and $\{2 \times 0\}$ respectively and hence can distinguish mirror images,

- $\Phi_{X}^{\beta}$ distinguishes the Square knot from the Granny knot with invariant values of $\left\{2 \times t+t^{2}\right\}$ and $\{2 \times 0\}$ respectively, so $\Phi_{X}^{\beta}$ is not determined by the knot group, and

- If we compute this invariant via the skein expansion rather than the state-sum method, it is important to freeze the diagram in place and not change any smoothed diagrams by Reidemeister moves, since these can change the value of the invariant.

\section{Quandle Brackets}

Let $X$ be a quandle, that is, a biquandle with $x \bar{\triangleright} y=x$ for all $x, y \in X$. An $X$-bracket in this case is called a quandle bracket.

Example 15. Consider the dihedral quandle $X$ on three elements, with operation table

$$
\left[\begin{array}{lll|lll}
1 & 3 & 2 & 1 & 1 & 1 \\
3 & 2 & 1 & 2 & 2 & 2 \\
2 & 1 & 3 & 3 & 3 & 3
\end{array}\right] .
$$

Then $X$-bracket over $\mathbb{Z}_{11}$ include $\beta$ given by

$$
\left[\begin{array}{lll|lll}
1 & 7 & 7 & 7 & 5 & 5 \\
1 & 1 & 8 & 7 & 7 & 1 \\
1 & 8 & 1 & 7 & 1 & 7
\end{array}\right]
$$


This is not a quandle 2-cocycle since $A_{x, y} \neq B_{x, y}$; our python code computed the following values for prime knots with up to eight crossings:

\begin{tabular}{r|l}
$\Phi_{X}^{\beta}(K)$ & $K$ \\
\hline 3 & $6_{2}$ \\
$3 u$ & $5_{2}, 7_{3}, 7_{6}, 8_{1}, 8_{2}$ \\
$3 u^{2}$ & $4_{1}$, \\
$3 u^{3}$ & $5_{1}, 8_{8}$ \\
$3 u^{5}$ & $7_{1}, 7_{5}, 8_{6}, 8_{12}$ \\
$3 u^{6}$ & $6_{3}, 8_{3}, 8_{14}$ \\
$3 u^{7}$ & Unknot, $3 u^{7}$ \\
$3 u^{9}$ & $7_{2}, 8_{7}, 8_{13}, 8_{17}$ \\
$3 u^{10}$ & $8_{9}, 8_{16}$ \\
$9 u$ & $8_{20}$ \\
$9 u^{2}$ & $8_{10}$ \\
$9 u^{3}$ & $8_{11}, 8_{15}$ \\
$9 u^{5}$ & $7_{4}, 8_{5}$ \\
$9 u^{7}$ & $8_{19}$ \\
$9 u^{8}$ & $7_{7}$ \\
$9 u^{10}$ & $8_{21}$ \\
$27 u^{2}$ & $8_{18}$.
\end{tabular}

Proposition 4. If $X$ is a quandle and $R$ is a commutative ring, then maps $A, B: X \times X \rightarrow R^{\times}$defining a quandle bracket must satisfy the mixed cocycle conditions

$$
\begin{array}{lll}
A_{x, y} A_{x \unrhd y, z} & =A_{x, z} A_{x \unrhd z, y \unrhd z} & (i) \\
A_{x, y} B_{x \unrhd y, z}=B_{x, z} A_{x \unrhd z, y \unrhd z} & (i i) \\
B_{x, y} A_{x \unrhd y, z}=A_{x, z} B_{x \unrhd z, y \unrhd z} & (\text { iii }) \\
B_{x, y} B_{x \unrhd y, z} & =B_{x, z} B_{x \unrhd z, y \unrhd z} & \text { (iv }) .
\end{array}
$$

Proof. Suppose our biquandle $X$ is a quandle, i.e., $x \bar{\triangleright} y=x$ for all $x, y \in X$. Then the first three biquandle bracket conditions from the Reidemeister III move reduce to

$$
\begin{aligned}
& A_{x, y} A_{y, z} A_{x \unrhd y, z}=A_{x, z} A_{y, z} A_{x \unrhd z, y \unrhd z} \quad A_{x, y} A_{x \unrhd y, z}=A_{x, z} A_{x \unrhd z, y \unrhd z} \\
& A_{x, y} B_{y, z} B_{x \unrhd y, z}=B_{x, z} B_{y, z} A_{x \unrhd z, y \unrhd z} \Rightarrow A_{x, y} B_{x \unrhd y, z}=B_{x, z} A_{x \unrhd z, y \unrhd z} \\
& B_{x, y} A_{y, z} B_{x \unrhd y, z}=B_{x, z} A_{y, z} B_{x \unrhd z, y \unrhd z} \quad B_{x, y} B_{x \unrhd y, z}=B_{x, z} B_{x \unrhd z, y \unrhd z}
\end{aligned}
$$

yielding (i),(ii) and (iv). Then the remaining biquandle bracket equations say

$$
\begin{aligned}
& A_{y, z}\left(A_{x, y} B_{x \unrhd y, z}-A_{x, z} B_{x \unrhd z, y \unrhd z}\right)=B_{y, z}\left(A_{x, z} A_{x \unrhd z, y \unrhd z}+\delta A_{x, z} B_{x \unrhd z, y \unrhd z}+B_{x, z} B_{x \unrhd z, y \unrhd z}\right) \\
& A_{y, z}\left(B_{x, y} A_{x \unrhd y, z}-B_{x, z} A_{x \unrhd z, y \unrhd z}\right)=-B_{y, z}\left(A_{x, y} A_{x \unrhd y, z}+\delta B_{x, y} A_{x \unrhd y, z}+B_{x, y} B_{x \unrhd y, z}\right)
\end{aligned}
$$

which then implies

$$
A_{y, z}\left(B_{x, y} A_{x \unrhd y, z}-A_{x, z} B_{x \unrhd z, y \unrhd z}\right)=\delta B_{y, z}\left(A_{x, z} B_{x \unrhd z, y \unrhd z}-A_{x, y} B_{x \unrhd y, z}\right)
$$

so we have

$$
\left(B_{x, y} A_{x \unrhd y, z}-A_{x, z} B_{x \unrhd z, y \unrhd z}\right)\left(A_{x, y}+\delta B_{y, z}\right)=0 .
$$

Then

$$
A_{y, z}+\delta B_{y, z}=A_{y, z}+\left(-A_{y z} B_{y, z}^{-1}-A_{y, z}^{-1} B_{y, z}\right) B_{y, z}=-A_{y, z}^{-1} B_{y, z}^{2}
$$

is a unit in $R$, so $B_{x, y} A_{x \unrhd y, z}-A_{x, z} B_{x \unrhd z, y \unrhd z}=0$ as required.

We note that the converse to proposition 4 is not true - the mixed cocycle conditions are necessary but not sufficient conditions for maps $A: X \times X \rightarrow R$ to define a quandle bracket, as the next example demonstrates. 
Example 16. Consider the trivial quandle on two elements, $T_{2}=\{1,2\}$ with $x \unrhd y=x \bar{\triangleright} y=x$. The maps $A, B: X \times X \rightarrow \mathbb{Z}_{3}$ defined by

$$
\left[\begin{array}{ll|ll}
1 & 1 & 1 & 1 \\
1 & 2 & 1 & 2
\end{array}\right]
$$

satisfy all four mixed cocycle conditions and also the conditions that

$$
\delta=-A_{x, y} B_{x, y}^{-1}-A_{x, y}^{-1} B_{x, y}=-2=1
$$

and

$$
w=2=A_{x, x} \delta+B_{x, x}
$$

for all $x, y \in X$; however, this is not a biquandle bracket since $A_{1,2} A_{2,2} B_{12}=2$ but

$$
A_{1,2} B_{2,2} A_{1,2}+A_{1,2} A_{2,2} B_{1,2}-2 A_{1,2} B_{2,2} B_{12}+B_{1,2} B_{2,2} B_{1,2}=4=1 \neq 2
$$

so the fourth equation in biquandle bracket axiom (iii) is not satisfied.

\section{Questions}

We end with some questions for future research. This is second paper in an ongoing series on quantum enhancements; future papers are underway extending the present results to knotted surface and virtual knots in various ways.

What exactly is the relationship between biquandle and brackets biquandle cohomology? Is there a generalized theory of biquandle cohomology which includes those biquandle brackets which are not biquandle cocycles in the traditional sense? Are there quantum enhancements which do not arise from biquandle brackets? What Khovanov homology-style categorifications of biquandle bracket invariants are possible? What about biquandle-colored skein modules?

\section{References}

[1] D. Bar-Natan. The knot atlas http://katlas.org/wiki/Main_Page.

[2] R. Bauernschmidt and S. Nelson. Birack modules and their link invariants. Commun. Contemp. Math., 15(3):1350006, 13, 2013.

[3] J. S. Carter, M. Elhamdadi, and M. Saito. Homology theory for the set-theoretic Yang-Baxter equation and knot invariants from generalizations of quandles. Fund. Math., 184:31-54, 2004.

[4] J. S. Carter, D. Jelsovsky, S. Kamada, L. Langford, and M. Saito. State-sum invariants of knotted curves and surfaces from quandle cohomology. Electron. Res. Announc. Amer. Math. Soc., 5:146-156 (electronic), 1999.

[5] J. Ceniceros, M. Elhamdadi, M. Green, and S. Nelson. Augmented biracks and their homology. Internat. J. Math., 25(9):1450087, 19, 2014.

[6] M. Elhamdadi and S. Nelson. Quandles - an introduction to the algebra of knots, volume 74 of Student Mathematical Library. American Mathematical Society, Providence, RI, 2015.

[7] R. Fenn, M. Jordan-Santana, and L. Kauffman. Biquandles and virtual links. Topology Appl., 145(13):157-175, 2004.

[8] R. Fenn, C. Rourke, and B. Sanderson. Trunks and classifying spaces. Appl. Categ. Structures, 3(4):321$356,1995$. 
[9] M. Graña. Quandle knot invariants are quantum knot invariants. J. Knot Theory Ramifications, 11(5):673-681, 2002.

[10] J. Hennig and S. Nelson. The column group and its link invariants. J. Knot Theory Ramifications, 21(7):1250063, 15, 2012.

[11] D. Hrencecin and L. H. Kauffman. Biquandles for virtual knots. J. Knot Theory Ramifications, 16(10):1361-1382, 2007.

[12] L. H. Kauffman and D. Radford. Bi-oriented quantum algebras, and a generalized Alexander polynomial for virtual links. In Diagrammatic morphisms and applications (San Francisco, CA, 2000), volume 318 of Contemp. Math., pages 113-140. Amer. Math. Soc., Providence, RI, 2003.

[13] W. B. R. Lickorish. An introduction to knot theory, volume 175 of Graduate Texts in Mathematics. Springer-Verlag, New York, 1997.

[14] S. Nelson and V. Rivera. Quantum enhancements of involutory birack counting invariants. J. Knot Theory Ramifications, 23(7):1460006, 15, 2014.

[15] S. Nelson and E. Watterberg. Birack dynamical cocycles and homomorphism invariants. J. Algebra Appl., 12(8):1350049, 14, 2013.

[16] M. Polyak. Minimal generating sets of Reidemeister moves. Quantum Topol., 1(4):399-411, 2010.

[17] S. Sawin. Links, quantum groups and TQFTs. Bull. Amer. Math. Soc. (N.S.), 33(4):413-445, 1996.

Department of Mathematical Sciences

Claremont McKenna College

850 Columbia Ave.

Claremont, CA 91711

Department of Mathematics

Harvey Mudd College

301 Platt Boulevard

Claremont, CA 91711 